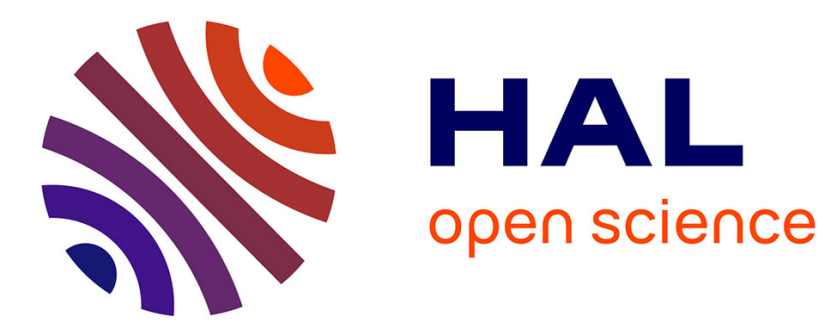

\title{
Découverte d'un bloc sculpté en bas-relief près du mausolée de Saint-Julien-lès-Martigues (Martigues, B.-du-Rh)
}

Lucien Rivet

\section{- To cite this version:}

Lucien Rivet. Découverte d'un bloc sculpté en bas-relief près du mausolée de Saint-Julien-lèsMartigues (Martigues, B.-du-Rh). Documents d'archéologie méridionale, 1987, 10, pp.120-123. 10.3406/dam.1987.991 . hal-01465142

\section{HAL Id: hal-01465142 \\ https://hal.science/hal-01465142}

Submitted on 5 Jan 2022

HAL is a multi-disciplinary open access archive for the deposit and dissemination of scientific research documents, whether they are published or not. The documents may come from teaching and research institutions in France or abroad, or from public or private research centers.
L'archive ouverte pluridisciplinaire HAL, est destinée au dépôt et à la diffusion de documents scientifiques de niveau recherche, publiés ou non, émanant des établissements d'enseignement et de recherche français ou étrangers, des laboratoires publics ou privés. 


\section{Découverte d'un bloc sculpté en bas-relief près du mausolée de Saint-Julien-lès-Martigues (Martigues, B.-du-Rh)}

\section{Lucien Rivet}

\section{Résumé}

Un bloc sculpté en calcaire de La Couronne a été découvert entre Martigues et Sausset-les-Pins à l'occasion de travaux routiers. Sans nul doute, cet élément architectural doit être rattaché au mausolée voisin de Saint-Julien, que l'auteur présente et illustre.

\section{Abstract}

A low-relief sculptured block found near the Saint-Julien-lès-Martigues mausoleum (Martigues, B.-du-Rb.).

A sculptured block carved out of La Couronne limestone was discovered during road works between Martigues and Sausset-lesPins. There is no doubt that this architectural component is linked to the near-by mausoleum at Saint-fulien, an Early Roman Empire monument presented and illustrated by the autor.

\section{Citer ce document / Cite this document :}

Rivet Lucien. Découverte d'un bloc sculpté en bas-relief près du mausolée de Saint-Julien-lès-Martigues (Martigues, B.-duRh). In: Documents d'Archéologie Méridionale, vol. 10, 1987. pp. 120-123;

doi : https://doi.org/10.3406/dam.1987.991

https://www.persee.fr/doc/dam_0184-1068_1987_num_10_1_991

Fichier pdf généré le 25/10/2018 


\title{
Découverte d'un bloc sculpté en bas-relief près du mausolée de Saint-Julien-lès-Martigues \\ (Martigues, B.-du-Rh.)
}

\author{
par Lucien RIVET *
}

Résumé - Un bloc sculpté en calcaire de La Couronne a été découvert entre Martigues et Sausset-les-Pins à l'occasion de travaux routiers. Sans nul doute, cet élément architectural doit être rattaché au mausolée voisin de Saint-Julien, que l'auteur présente et illustre.

(Mots-clés : Sculpture, Mausolée, Haut-Ëmpire romain, Saint-Julien-lès-Martigues)

\section{A low-relief sculptured block found near \\ the Saint-Julien-lès-Martigues mausoleum (Martigues, B.-du-Rb.)}

\begin{abstract}
A sculptured block carved out of La Couronne limestone was discovered during road works between Martigues and Sausset-les-Pins. There is no doubt that this architectural component is linked to the near-by mausoleum at Saint-Julien, an Early Roman Empire monument presented and illustrated by the autor.
\end{abstract}

(Key words : Sculpture, Mausoleum, Early Roman Impire, Saint-Julien-lès-Martigues)

\section{La découverte}

Au cours du printemps 1987 furent engagés des travaux d'élargissement de la route départementale $n^{\circ} 5$ entre Martigues et Sausset-les-Pins. En mars 1987, nous prospections entre les Ventrons et Saint-Julienlès-Martigues les travaux qui touchaient exclusivement le flanc sud de l'ancien tracé ; c'est durant ce parcours que fut découvert le bloc dont il est question ici (1), à l'angle nord-ouest d'un champ, près d'un ruisseau intermittent (2).

Il s'agit d'un bloc en pierre de La Couronne (3) (Rivet 1979a ; Guéry 1981), de forme parallélépipédique $(1,40 \times 0,51 \mathrm{~m}$ de $\mathrm{H}$. $\mathrm{x}$ $0,43 / 0,45 \mathrm{~m}$ d'ép.). Hormis la face principale, qui est sculptée, les cinq autres côtés sont seulement dégrossis, mais on note les trois particularités suivantes:

- à la base du flanc droit, de même qu'au sommet du flanc gauche, un méplat d'une dizaine de centimètres de haut a été aménagé, d'un côté en léger retrait du bossage, de l'autre en faible saillie, ; il s'agit d'un aménagement pour recevoir, de part et d'autre de ce bloc et à sec, d'autres pierres semblables ;

- sous l'arête inférieure gauche se trouve une entaille régulière (longue de $20 \mathrm{~cm}$, large de 4 à $5 \mathrm{~cm}$ et profonde ded $8 \mathrm{~cm}$ ) dont on ne saisit pas clairement l'utilité (trou de levage, de manutention, de scellement ?) ;

- sur la face supérieure et sensiblement au milieu est conservé un trou $(8 \times 3 \mathrm{~cm})$, vraisemblablement de levage.
La face principale est ornée d'une sculpture en bas-relief (fig. 1), mais seule la partie gauche conserve une figuration bien visible, la partie droite étant dégradée, ou, plus vraisemblablement, non décorée. Aux divers endroits où le décor est le moinsd abîmé, le relief a une épaisseur qui varie entre 4,5 et $8 \mathrm{~cm}$.

Le relief présente un buste de personnage, de face, portant un vêtement plissé; l'usure de la pierre ne permet pas de distinguer d'autres détails sauf, ici ou là, les traces laissées par l'emploi du trépan pour le traitement du vêtement ainsi que pour les contours du personnage. Ce buste est encadré de deux têtes et encolures de chevaux qui regardent vers la droite. Le bras gauche de l'homme, replié devant sa poitrine, est tendu vers le museau du cheval de gauche, sans doute pour le tenir par le mors.

\section{Les vestiges du mausolée de Saint-Julien}

Le mur nord de la chapelle de Saint-Julien-lèsMartigues présente, à partir de trois assises de blocs, un panneau sculpté de 3,80 x 1,50 m (fig. 2 et 3). 


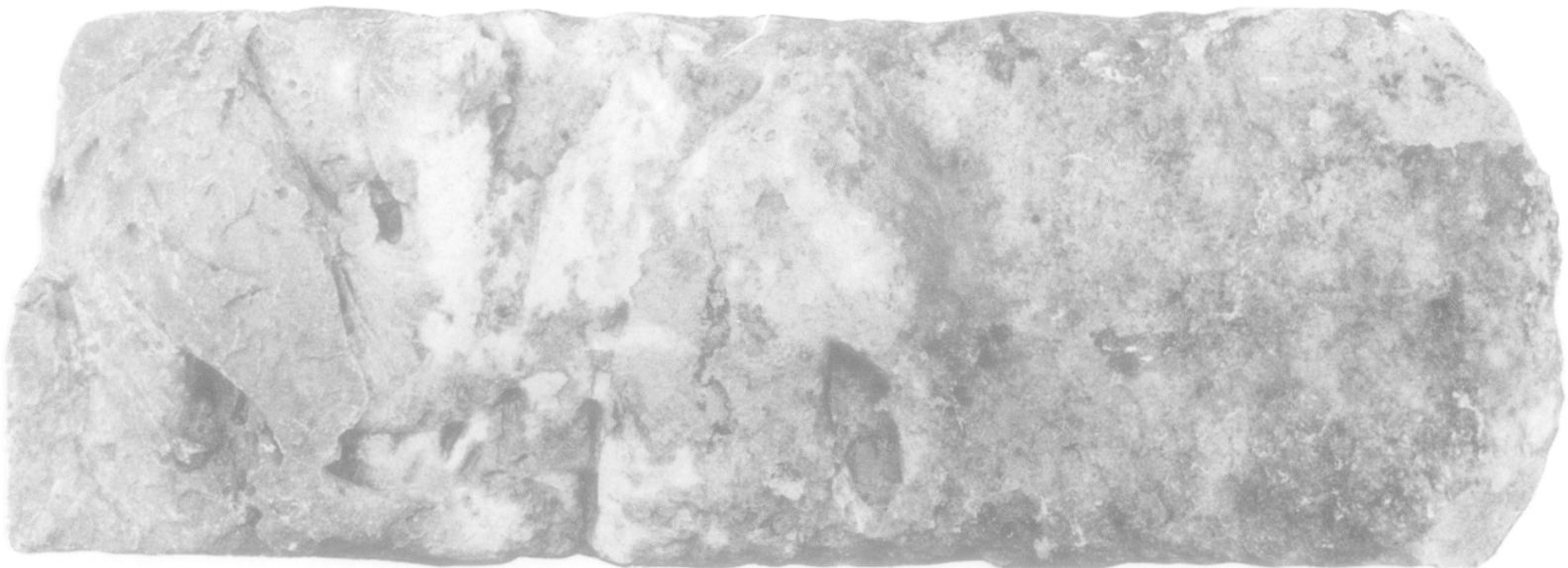

1 Ie bloc sculpté en bas-relief, découvert en 1987 (cl. auteur).

La scène conservée comporte huit personnages. Au centre, assis de part et d'autre de l'axe de symétrie, un homme et une femme ; deux silhouettes, debout, sont entre eux et à l'arrière-plan, tandis que deux hommes, en pieds, revêtus de la toge, les encadrent. Vient ensuite, pour chaque côté, un autre homme debout : celui de droite, dont le corps est revêtu d'une cotte de maille, se tient près d'un cheval (dont on ne distingue qu'une patte antérieure).

Cette représentation a donné lieu à des commentaires parfois sensiblement divergents (Espérandieu
1907, 104 ; Benoit 1936, n 153 ; Gérin-Ricard 1939 ; Picard 1943 ; Benoit 1948 ; Rivet 1979b) (4), mais l'interprétation globale renvoie toujours, sans que cela soit contradictoire, à une image à la fois familiale et funéraire. La controverse a plutôt porté sur un hypothétique déplacement des vestiges de ce monument; nul doute pourtant qu'il est en place et qu'il a servi d'appui aux différents états constitutifs de la chapelle actuelle, le soubassement et le socle sculpté de la face nord ayant seuls survécus aux récupérations des différents chantiers de construction. Quant à l'interpréta-

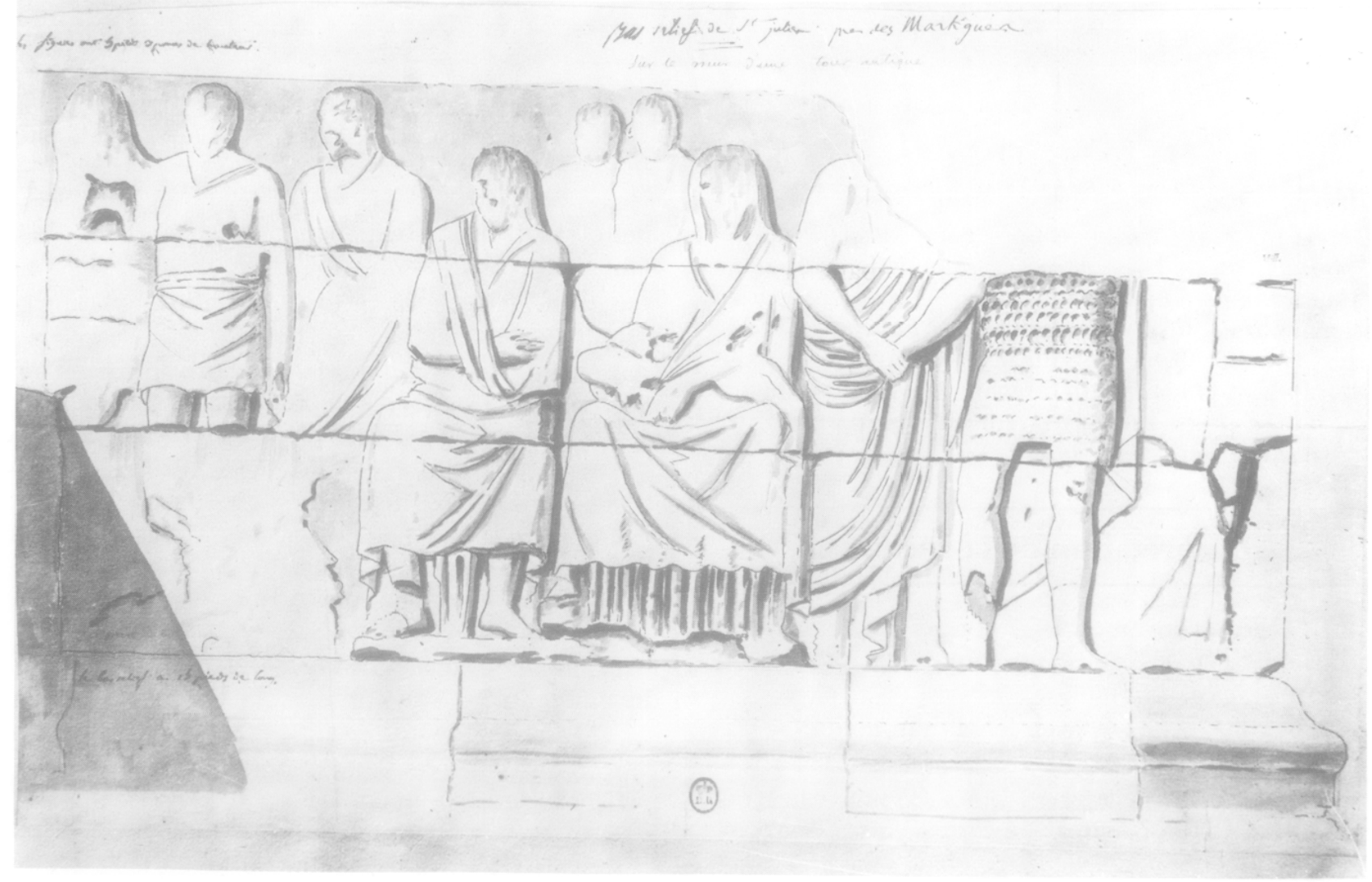

2 Le panneau sculpté du mausolée de Saint-Julien-lès-Martigues en 1811 : dessin d'Aubin Louis Millin (Biblio. Nat., VA 13.T.9). 


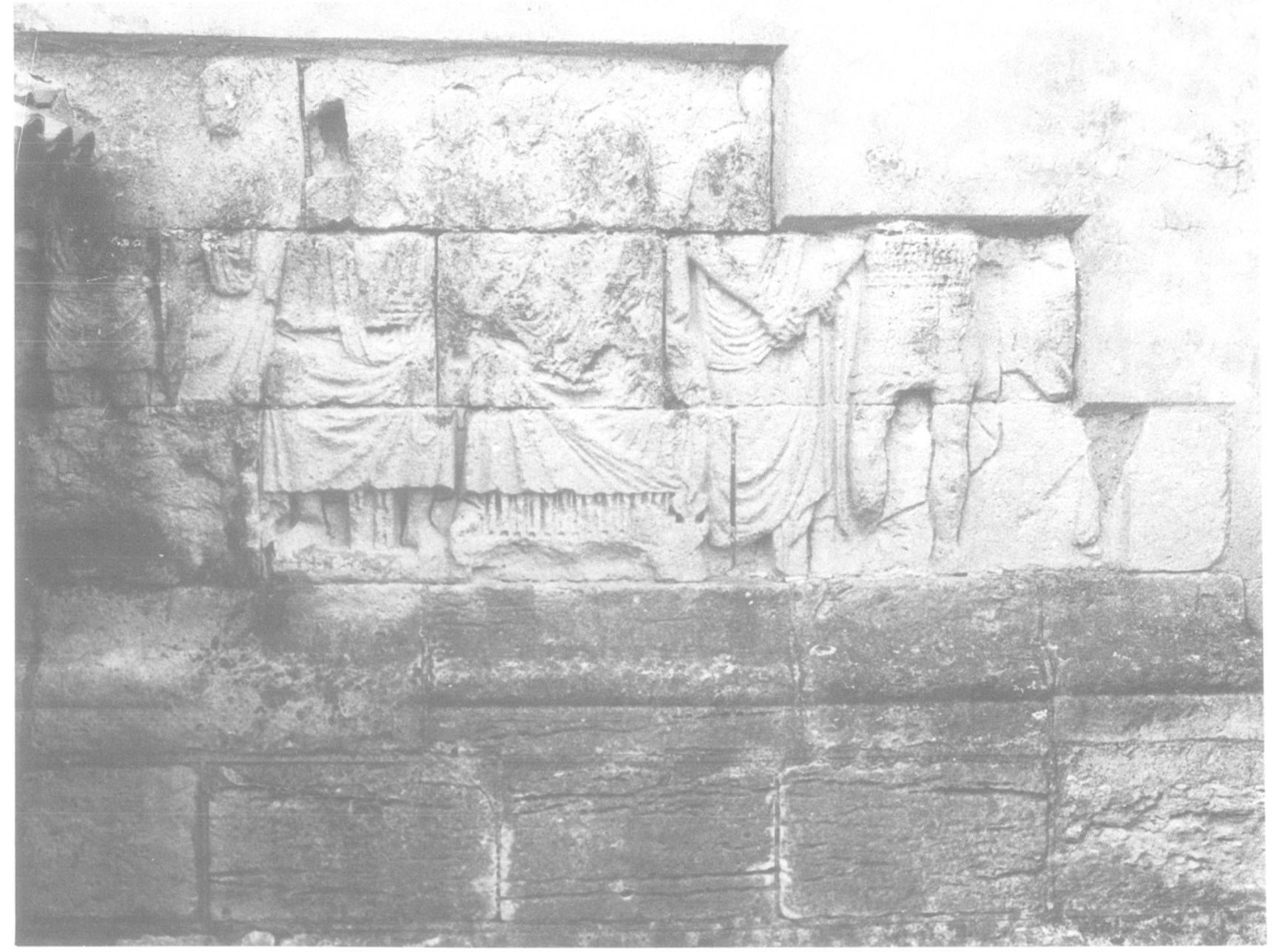

3 Le panneau sculpté de Saint-Julien-lès-Martigues : état en 1982 (cl. auteur).

tion du monument, elle ne pose guère de difficultés : le soubassement, la qualité et le sens de la sculpture, les dimensions du panneau conservé, renvoient tous à un mausolćc du type de celui de Glanum (Rolland 1969 ; Bruchet 1969) (5). Sous réserve d'une étude plus approfondic, la réalisation du mausolée de Saint-Julien pourrait se situer autour des années qui marquent le changement d'ère.

\section{Interprétation de la découverte}

Le bloc découvert en 1987 et ceux qui composent le bas-relief du mausolée de Saint-Julien présentent plusieurs points communs :

- au plan du matériau, avec le calcaire tendre de La Couronne ;

- au plan des dimensions des blocs ; le nouveau bloc mesure $0,51 \mathrm{~m}$ de hauteur et les trois assises du socle du mausolée mesurent, de bas en haut : 0,53;0,51 et $0,51 \mathrm{~m}$; pour la longueur de $1,40 \mathrm{~m}$, la mesure s'inscrit également dans les dimensions que l'on peut réaliser sur trois blocs intacts du panneau conservé : 1,$26 ; 1,46$ et $1,53 \mathrm{~m}$;

- au plan de l'échelle de représentation, quasiment grandeur naturelle ;

- au plan de l'organisation du relief qui se répartit sur trois assises, l'assise supérieure (dans l'état actuel) ćtant réservée à la représentation des têtes et des torses. Notre bloc correspondrait donc au niveau de la troisième assise sculptée ;

- au plan de la qualité du relief avec l'utilisation de la technique du "cerne" qui consiste à détourer, au trépan, sur une profondeur de un à deux centimètres, les contours des bas-reliefs (6).

Rien, en revanche, ne permet d'observer la moindre différence.

Chacun de ces points apparente donc le bloc découvert en 1987 à ceux qui constituent les vestiges du mausolée de Saint-Julien, qui ne conserve qu'une de ses quatre faces, les trois autres ayant totalement disparu de l'architecture de la chapelle. Nul doute que ce nouveau bloc lui appartienne malgré la distance $(1700 \mathrm{~m})$ de son lieu de découverte. 


\title{
Notes de commentaire
}

* C.N.R.S., Centre Camille Jullian - 13621 AIX-EN-PROVENCE:

1 - Les terrassements nécessitaient un approfondissement (plus rarement un remblaicment) de la margc sud de l'ancien tracé. La prospection consistait à observer les terres remuées et les coupes éventuelles provoquées par les engins mécaniques. Partout où il y eut approfondissement, les engins constituèrent, parallèlement, un talus de terre ; le bloc, soulevé par un engin, avait roulé au sud de ce talus.

2 - Au lieu-dit "Les Serens", quartier "Les Plaines". Cadastre : section DM, parcelle 31 (angle nord-ouest); coordonnées Lambert III : 822,$25 ; 122,60$. Prospection du 14 mars 1987.

3 - Les lieux d'extraction se situent à trois ou quatre kilomètres de Saint-Julien.

4. La tradition s'est évertuée, on ne sait pour quelle(s) raison(s), et sans aucun argument, à démontrer que l'assemblage de ces blocs n'était pas en place.

5 - La mesure de la largeur du panneau de Saint-Julien doit être de 4,20 ou 4,30 m, d'après l'axe de symétrie. Pour le mausolée de Glanum, la mesure est de 4,36/4,41 m.

6 - Cette technique est commune à un certain nombre de bas-reliefs qui parent des monuments de la rive orientale de la basse

\section{Renvois bibliographiques}

Benoit 1936 : BENOIT (F.) - Carte archéologique de la Gaule romaine (F.O.R.). Paris, V, 1936, 232 p., VII pl., 1 carte.

Benoit 1948 : BENOIT (F.) - Le bas-relief de Saint-Julien-lès-Martigues (B.-du-Rh.). Gallia, VI, 1948, pp. 171-175.

Bruchet 1969 : BRUCHET (J.) et RIGOIR (Y.) - Les Antiques. Gap, 1969, 88 p. (La Provence archéologique et médiévale, 1).

Espérandieu 1907 : ESPERANDIEU (E) - Recueil général des basreliefs de la Gaule. Paris, I, 1907.

Gérin-Ricard 1939 : GERIN-RICARD (H. de)-Notes d'Archéologie. Provincia, 1939, pp. 93-98.

Guéry 1981 : GUERY (R.), PIRAZZOLI (P.) et TROUSSET (P.) - Les carrières littorales de La Couronne, indices de variation du niveau marin. Dossiers $H$ ist. el Archécl., 50, 1981, pp. 18-27.
Picard 1943 : PICARD (Ch.) - Le bas-relief de Saint-Julien-lèsMartigues. C. R. de l'Acad. Inscr. Belles Lett., 1943, pp. 439-458.

Rivet 1973-4 : RIVET (L.) - La sculpture gallo-romaine en Arles. Notes à propos de douze sculptures inédites. Cab. Lig. Préb. Archéol, 22-23, 1973-1974, pp. 239-262.

Rivet 1979a : RIVET (L.) - Les carrières de La Couronne. In : Guide archéologique des rives de l'étang de Berre. Aix-en-Provence, Edisud, 1979, pp. 43-45.

Rivet 1979b : RIVET (L.) - Le bas-relief de Saint-Julien-lèsMartigues. In: Guide archéologique des rives de l'étang de Berre. Aix-en-Provence, Edisud, 1979, pp. 64-65.

Rolland 1969 : ROLLAND (H.) - Le mausolée de Glanum. Paris, éd. du C.N.R.S., 1969 (suppl. à Gallia, XXI).

\section{Un lot de Dérivées-des-Sigillées Paléochrétiennes à Iluro (Mataró, El Maresme, Espagne)}

\author{
par Albert Bacaria i Martrus *
}

Résumé - Les fouilles récentes de Mataró (Catalogne, Espagne) viennent de mettre en évidence, dans un niveau scellé par un pavement, un lot de céramiques Dérivées-des-Sigillées Paléochrétiennes dont une assiette de forme Rigoir 1 richement ornée. L'intérêt de la présentation réside dans la datation du contexte : la couche concernée parait être mise en place peu après 425 . Cette note contribue à la connaissance des importations de ces céramiques (essentiellement languedociennes) dans le nord-est de la Péninsule ibérique.

(Mots-clés : Céramiques Dérivées-des-Sigillées Paléochrétiennes, Chronologie, Mataró, Espagne) 